\title{
0637 - ASSOCIATION OF DIABETIC NEPHROPATHY AND RETINOPATHY WITH ABNORMAL LIPID PARAMETERS - A DYSLIPIDEMIC DIABETIC COMPLEX?
}

Shakeel, S.(1)*;Khan, M.I.H.(2);Tayyab, G.U.N.(3);Ehsan Ullah, H.(4);Zulfiqar, M.(5) Post Graduate Medical Institute/AMC/Lahore General Hospital, Lahore, Pakistan
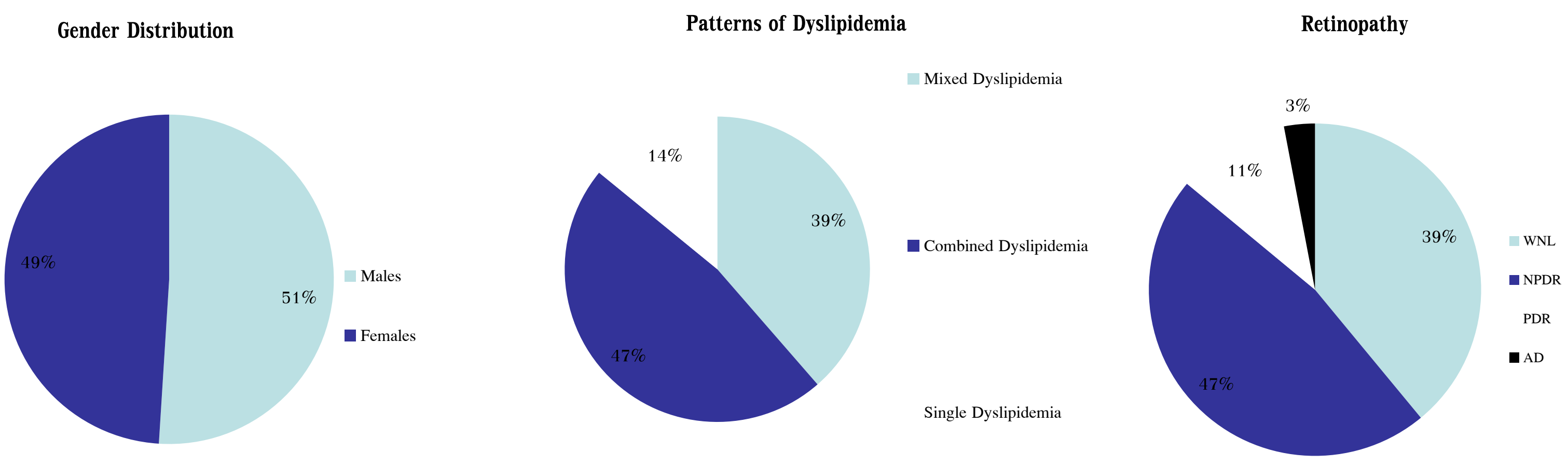

\section{Background:}

Diabetic Dislipidus or Diabetic Dyslipidemia is a major cause of cardiovascular morbidity and mortality. Poor glycemic control can lead to blindness and renal failure. However, this relationship between uncontrolled blood sugar, abnormal lipid parameters, retinopathy and nephropathy has not been studied previously.

\section{Aim:}

This study was conducted to find out the association of Poor glycemic levels in Type II Diabetics having Nephropathy, Retinopathy and Dyslipidemia.

\section{Methods:}

It was a single centered, retrospective analysis of 572 Diabetics T2DM patients who were brought to the Diabetes, Endocrine \& Metabolic Centre (DEMC) of Lahore General Hospital (LGH), Lahore from January 2016 to December 2016 for routine checkup. The patients with (HbA1c>9\%) were identified as candidates with poor glycemic control and were further studied. Their fasting serum levels for Total Cholesterol (TC), Low Density Lipoproteins (LDL), High Density Lipoproteins (HDL) and Triglyceride (TG) were checked and the above patients were labeled as Mixed dyslipidemia ( $>2$ abnormal lipid parameters), combined dyslipidemia (at least 2 abnormal lipid parameters) and single dyslipidemia (only 1 abnormal lipids). Furthermore, their urinary microalbumin levels (spot urine) were assessed qualitatively and Fundus Photograph findings were noted for diabetes related changes, if present. These readings were correlated and analyzed by using Statistical Package of Social Sciences (SPSS) Version 20 and descriptive statistics were recorded in the form of mean and percentages.
Results:

\begin{tabular}{|c|c|c|}
\hline CATEGORY & MEAN & SD \\
\hline AGE & 43.17 & \pm 9.83 Years \\
\hline $\mathrm{HbA1c}$ & 10.07 & $\pm 1.06 \%$ \\
\hline $\mathrm{TC}$ & 199.1 & $\pm 51.79 \mathrm{mg} / \mathrm{dl}$ \\
\hline TG & 183 & $\pm 79 \mathrm{mg} / \mathrm{dl}$ \\
\hline LDL & 127.83 & \pm 41.72 \\
\hline HDL & 41.20 & $\pm 9.78 \mathrm{mg} / \mathrm{dl}$ \\
\hline \multicolumn{3}{|c|}{ Nephropathy } \\
\hline & & $\begin{array}{l}\square \text { Microalbumin (pos) } \\
\text { - Microalbumin (neg) }\end{array}$ \\
\hline
\end{tabular}

\section{Conclusion:}

Higher HbA1c levels indicating poor glycemia show strong association with Nephropathy, Retinopathy and Dyslipidemia. It can be grouped as "Dyslipedemic Diabetic Complex" requiring multidisciplinary approach for management and to prevent cardiovascular complications. 